\title{
Síndrome Gorham-Stout - um desafio diagnóstico e terapêutico
}

\section{Gorham-Stout syndrome - a diagnostic and therapeutic challenge}

Fernando Lopes Ponte Neto ${ }^{1,2}$. Daniel Aguiar Dias². Lucas Mustafa Aguiar². Karine Sampaio Sena². Gabriela Studart Galdino ${ }^{1}$.

1 Universidade Federal do Ceará (UFC), Fortaleza, Ceará, Brasil. 2 Hospital Universitário Walter Cantídio (HUWC), Fortaleza, Ceará, Brasil.

\section{RESUMO}

A linfangiomatose difusa, também conhecida como síndrome Gorham-Stout, é uma doença rara. A patologia causa alterações morfológicas devido a infiltração de tecido linfático, além da formação de derrame pleural quiloso e de ascite quilosa, podendo acometer diversos órgãos como ossos, baço, pulmão e outros. É ainda considerada um desafio diagnóstico devido ao pouco conhecimento sobre a doença, o número limitado de casos relatados, e devido ao acometimento difuso, com diversas possibilidades de manifestações clínicas a depender do órgão acometido.

O padrão ouro para diagnóstico continua sendo o anátomo-patológico, porém, achados radiológicos sugestivos, juntamente com clínica compatível e a comprovação de derrames quilosos, tendo outras etiologias afastadas, confirmam diagnóstico presuntivo de linfangiomatose difusa. Até o momento, nenhuma terapêutica se mostrou eficaz, sendo uma doença com evolução desfavorável e prognóstico ruim. $\mathrm{O}$ estudo em questão relata um caso de linfangiomatose difusa presumida.

Palavras-chave: Linfangioma. Malformações vasculares. Quilotórax.

\section{ABSTRACT}

Diffuse lymphangiomatosis, also known as Gorham-Stout syndrome, is a rare disease. The pathology causes morphological alterations caused by the infiltration of lymphatic tissue, as well as the formation of chylous pleural effusion and chylous ascites, which can affect several organs such as bones, spleen, lung and others. Still considered a diagnostic challenge due to the limited knowledge about the disease, the limited number of cases reported, and due to diffuse involvement, with several possibilities of clinical manifestations depending on the affected organ.

The gold standard for diagnosis remains the anatomo-pathological, but suggestive radiological findings, along with compatible clinical and evidence of chylous effusions, having other etiologies away, confirm a presumptive diagnosis of diffuse lymphangiomatosis. To date, no therapy has been effective, being a disease with unfavorable evolution and poor prognosis. The present study reports a case of presumed diffuse lymphangiomatosis.

Keywords: Lymphangioma. Vascular malformations. Chylothorax.

Autor correspondente: Fernando Lopes Ponte Neto, Rua Izabel Negrão Bertotti, 101, Mansões Santo Antônio, Campinas, São paulo. CEP: 13087508. Telefone: +5588 99644-8193. E-mail: fernandopontemed@gmail.com

Conflito de interesses: Não há qualquer conflito de interesses por parte de qualquer um dos autores.

Recebido em: 17 Mar 2019; Revisado em: 26 Abr 2020; Aceito em: 26 Abr 2020. 


\section{INTRODUÇÃO}

A linfangiomatose difusa, também conhecida como Gorham-Stout, é uma doença rara, ${ }^{1}$ mais incidente em crianças e adultos jovens, ${ }^{2}$ caracterizada pela proliferação anômala de canais vasculares e linfáticos. ${ }^{3}$ As causas desta patologia ainda não são totalmente definidas, ${ }^{4}$ contudo, acredita-se que a etiologia seja multifatorial..$^{5}$ A apresentação clínica é bastante variável e depende dos órgãos acometidos. A maior parte dos casos apresentam comprometimento pulmonar e ósseo caracterizado por lesões osteolíticas, sendo possível ainda acometimento hepático, esplênico e cutâneo., ${ }^{5,6} \mathrm{~A}$ linfangiomatose é uma condição marcada pelo aumento no tamanho e número de canais linfáticos de paredes finas que são interligados e dilatados de forma patológica formando cistos. $^{7}$ As complicações dessa entidade são decorrentes das confluências desses canais e cistos que tendem a infiltrar os tecidos circundantes, causando alterações devido a invasão e/ ou compressão de estruturas, apesar da ausência de malignidade histológica ou infecção. ${ }^{5}$ Entre as possíveis complicações destacam-se como mais graves: quilotórax e a compressão de medula espinhal. ${ }^{5}$ Apresentando pouco mais de 200 casos relatados, ${ }^{5}$ por ser extremamente rara é pouco diagnosticada e não se tem dados precisos quanto à real prevalência da síndrome. Sua natureza incomum a torna a um desafio diagnóstico e terapêutico. ${ }^{8}$ Objetivou-se relatar um caso de linfangiomatose difusa - Síndrome de Gorham-Stout do Hospital Universitário Walter Cantídio da Universidade Federal do Ceará.

\section{RELATO DO CASO}

Homem de 24 anos previamente hígido apresentou quadro de dor em região retroesternal, agravada com a inspiração profunda. Houve resolução espontânea, mantendo-se assintomático durante 2 anos, até surgimento de linfonodomegalias em região inguinal à direita, menor que $1(\mathrm{um}) \mathrm{cm}$, dolorosa, sem crescimento aparente, ou sinais que sugerissem malignidade. Concomitantemente iniciou quadro de dispneia aos grandes esforços, com progressiva sensação de peso em região torácica. Queixava ainda de quadro álgico em região abdominal (hipocôndrio direito e fossa ilíaca direita), tórax e região lombar de intensidade variada, sem fator desencadeante, porém, com piora à movimentação e melhora parcial com analgésicos. Quadro abdominal era acompanhando por equimose e edema local que duravam poucos dias, associado a dor à compressão e sem associação com sintomas dispépticos. Apresentou perda ponderal de $8 \mathrm{~kg}$ (15\% do peso habitual) em 7 meses. Negava febre, tosse, dispneia ao repouso, edema de membros, alterações do hábito evacuatório ou lesões de pele.

Em investigação inicial, evidenciou ao exame físico derrame pleural e ascite. O estudo radiológico mostrou alterações heterogêneas em vértebras e cistos esplênicos. Durante internação não chegou à completa elucidação etiológica do quadro, e mesmo sem confirmação diagnóstica realizou tratamento empírico para tuberculose pleural com rifampicina, isoniazida, pirazinamida e etambutol, com aparente melhora do quadro. Porém, após 6 meses de tratamento regular, paciente mantinha queixas de dor torácica que agravava com inspiração profunda e esporádica dispneia em repouso, além de desconforto abdominal equivalente a dor do início do quadro. Paciente novamente foi submetido a toracocentese guiada por ultrassonografia. O líquido puncionado apresentava aspecto quilo-hemático, sendo confirmado após análise laboratorial, aumento na dosagem de triglicerídeos da amostra coletada (195mg/dL-referência: $110 \mathrm{mg} / \mathrm{dL})$, e quantidade insignificante de hemácias. A investigação foi complementada com estudo radiológico avançado com tomografia computadorizada e ressonância nuclear magnética. Paciente foi submetido à biópsia de vertebra, sem intercorrências, porém, com laudos histopatológicos inconclusivos, visto que o material coletado não caracterizou a lesão, sendo formado somente por tecido ósseo sem alteração.

Paciente negava uso ativo de álcool, tabaco e substâncias psicoativas. Morava em casa de alvenaria; em boas condições hidrossanitárias, não criava animais. Negava história familiar equivalente ao quadro. Nega consanguinidade dos pais.

O paciente relatado apresentava queixas vagas, com acometimento de diversos órgãos, no qual a principal queixa foi dor (abdome, tórax, coluna lombar), seguida por quadro dispneico e sensação de peso no peito, sendo posteriormente diagnosticado derrames pleurais e ascite. O diagnóstico foi estabelecido visto quadro clínico compatível, além da confirmação de derrame pleural quiloso (quilotórax) e, sobretudo, pelas alterações radiológicas características, como formações císticas, derrames cavitários e lesões ósseas. O paciente seguiu ambulatorialmente com queixas de dor abdominal discreta, dispneia e manutenção do derrame pleural bilateral, sendo optado por iniciar propranolol.

\section{Resultado dos Principais Exames Complementares:}

- Ultrassonografia (USG) abdominal: esplenomegalia com múltiplas formações císticas no parênquima esplênico (microabcessos, microbacteriose, doença fúngica) e ascite.

\section{- USG de tórax: derrame pleural volumoso bilateral}

- Ressonância magnética (RM) de pelve e coluna lombo-sacra: alteração difusa do sinal da medula óssea da coluna e da pelve, destacando-se áreas mal definidas com hipersinal na sequência T1, além de alterações focais alongadas, tortuosas, por vezes confluentes, de aspecto cístico, com alto sinal na sequência T2 e hiposinal T1, as quais exibem realce nas fases pós-contraste, mais evidentes no corpo vertebral de L1 e na asa do íliaco direito. Destaca-se acometimento também dos arcos costais, os quais se apresentam expandidos. Impressão diagnóstica: Alterações de aspecto cístico de todas as estruturas do esqueleto axial. Volumoso derrame pleural bilateral, ascite, formações de aspecto cístico na região perirretal e no baço.

- Tomografia Computadorizada (TC) de tórax: coleções líquidas extrapleurais bilateralmente, mais volumosa à direita. Existe conteúdo gasoso em permeio à coleção situada à direita, 
estendendo-se ao mediastino, aos planos músculos adiposos da parede torácica, inclusive para a cavidade medular de arco costal adjacente. Dentro do contexto clínico atual, sugere-se considerar a possibilidade de malformações linfáticas (linfangiomas). Derrame pleural de moderado volume bilateral. Sinais de infiltração patológica da medula óssea do esqueleto axial, devendo representar linfangiomas dentro da hipótese clínica aventada (anomalia linfática generalizada/ linfangiomatose generalizada).

\section{DISCUSSÃO}

Os sintomas presentes na síndrome de Gorham-Stout dependem dos sistemas de órgãos envolvidos e da extensão acometida. No início da doença, os pacientes podem ser assintomáticos devido ao curso evolutivo lento da enfermidade, quando surgem, os sintomas são inespecíficos para a síndrome, assim, os pacientes podem ser conduzidos de forma imprecisa. ${ }^{6}$ Os sinais torácicos são dispneia, dor torácica, tosse, sibilânica, derrame pleural, dor óssea, fraturas patológicas e deformidades torácicas. ${ }^{9}$ Além disso, a ocorrência de derrames quilólicos é achado importante na suspeita diagnóstica. ${ }^{10}$ Quadro abdominal com dor e distensão são referidos no acometimento intestinal e peritoneal. $\mathrm{O}$ diagnóstico se torna um desafio devido à raridade e amplo espectro de características clínicas, histológicas e de imagem. Uma pista diagnóstica importante é a coexistência de lesões ósseas líticas ${ }^{6}$ e derrames quilosos (pleural e/ou ascítico). ${ }^{4}$ Os achados na tomografia computadorizada de tórax que podem favorecer o diagnóstico ${ }^{3,10}$ incluem o seguinte: “(I) espessamento bilateral dos septos interlobulares e feixes broncovasculares; (II) opacidades irregulares de vidro fosco; (iii) infiltração líquida difusa no tecido mole mediastinal e hilar, resultante da proliferação difusa de canais linfáticos e acumulação de líquido linfático; e (iv) derrame de pleura bilateral".? Os achados radiológicos abdominais incluem confluência de lesões de baixa densidade, parênquima esplâncnico heterogêneo com presença de lesões císticas e espessamento mesentérico pode ser visto em alguns casos. ${ }^{11}$

\section{REFERÊNCIAS}

1. Witte MH. Lymphangioleiomyomatosis and Gorham-Stout disease: primary or secondary disorders of the lymphatic system? Lymphology. 2017;50(3):114-19.

2. Faul JL, Berry GJ, Colby TV, Ruoss SJ, Walter MB, Rosen GD, et al. Thoracic lymphangiomas, lymphangiectasis, lymphangiomatosis and lymphatic dysplasia syndrome. Am J Respir Crit Care Med. 2000;161(3 pt 1):1037-46.

3. Lim HJ, Han J, Kim HK, Kim TS. A rare case of diffuse pulmonary lymphangiomatosis in a middle-aged woman. Korean J Radiol. 2014;15(2):295-9.

4. Lima AS, Martynychen MG, Florêncio RT, Rabello LM, Barros JA, Escuissato DL. Pulmonary lymphangiomatosis: a report of two cases. J Bras Pneumol. 2007;33(2):229-33.

5. Johnstun J, Brady L, Simstein R, Duker N. Chronic recurrent
Cutillo et al descreveram que a linfangiomatose também pode envolver outras vísceras abdominais e o retroperitônio. ${ }^{12}$ A biópsia com análise anatomopatológica continua sendo a ferramenta de diagnóstico definitivo; ${ }^{1-8}$ entretanto, estabelece como possível diagnóstico preciso o caso com sintomatologia, derrames quilosos e padrão radiográfico compatível. ${ }^{13}$

Não existe abordagem terapêutica padrão., ${ }^{3,10}$ Por vezes, a mesma visa paliativamente ${ }^{6}$ reduzir os sintomas, visto a ausência de cura para a síndrome. Entre as opções já consideradas se tem: transfusão de albumina, dietas de triglicerídeos de cadeia média com baixo teor de gordura, interferon alfa, corticosteroides, tamoxifeno, bisfosfonatos, imatinib, talidomida, ciclofosfamida, acetato de medroxiprogesterona, octreotide, vincristina e ligação do ducto torácico para o quilotórax, ${ }^{8,14,15}$ porém, até o momento sem resultados promissores e com efeitos adversos importantes. Recentemente outras terapêuticas vêm sendo testadas, entre elas, o tratamento com propranolol, sirolimus e bevacizumab. ${ }^{14,15}$

O propranolol vem sendo usado como alternativa terapêutica em patologias que visam o controle da proliferação angiogênica, vista ação na redução da via de sinalização da proteína quinase ativada da RAF que reduz VEGF, além de indução à apoptose de células endoteliais capilares. $\mathrm{O}$ fator de crescimento vascular (VEGF) vem sendo associado a proliferação linfática, visto a expansão do seu papel para além de fator angiogênico, sendo considerado linfangênico. Assim, estudos avaliam o propranolol como possível agente alvo para a terapia de controle da linfangiomatose. ${ }^{14,15}$

\section{CONSENTIMENTO}

Foi obtido consentimento livre esclarecido assinado pelo paciente.

\section{DECLARAÇÃO DE CONFLITO DE INTERESSE}

Nenhum.

Gorham-Stout syndrome with cutaneous involvement. Rare Tumors. 2010;2(3):e40.

6. Joshi M, Phansalkar DS. Simple lymphangioma to generalized lymphatic anomaly: role of imaging in disclosure of a rare and morbid disease. Case Rep Radiol. 2015;2015:603859.

7. Du MH, Ye RJ, Sun KK, Li JF, Shen DH, Wang J, et al. Diffuse pulmonary lymphangiomatosis: a case report with literature review. Chin Med J (Engl). 2011;124(5):797-800.

8. Ellati R, Attili A, Haddad H, Al-Hussaini M, Shehadeh A. Novel approach of treating Gorham-Stout disease in the humerus - case report and review of literature. Eur Rev Med Pharmacol Sci. 2016;20(3):426-32.

9. Wong CS, Chu TY. Clinical and radiological features of generalised lymphangiomatosis. Hong Kong Med J. 2008;14(5):402-4. 
10. Kadakia KC, Patel SM, Limper AH, Limper AH. Diffuse pulmonary lymphangiomatosis. Can Respir J. 2013;20(1):52-4.

11. Wunderbaldinger P, Paya K, Partik B, Turetschek K, Hörmann M, Horcher E, et al. CT and MR imaging of generalized cystic lymphangiomatosis in pediatric patients. AJR Am J Roentgenol. 2000;174(3):827-32.

12. Cutillo DP, Swayne LC, Cucco J, Dougan H. CT and MR imaging in cystic abdominal lymphangiomatosis. J Comput Assist Tomogr. 1989;13(3):534-6.
13. Pyatt RS, Williams ED, Clark M, Gaskins R. Case report. CT diagnosis of splenic cystic lymphangiomatosis. J Comput Assist Tomogr. 1981;5(3):446-8.

14. Hangul M, Kose M, Ozcan A, Unal E. Propranolol treatment for chylothorax due to diffuse lymphangiomatosis. Pediatr Blood Cancer. 2019;66(5):e27592.

15. Ozeki M, Fukao T, Kondo N. Propranolol para linfangiomatose difusa intratável. N Engl J Med. 2011;364(14):1380-2.

\section{Como citar:}

Ponte FL Neto, Dias DA, Aguiar LM, Sena KS, Galdino GS. Síndrome Gorham-Stout - um desafio diagnóstico e terapêutico. Rev Med UFC. 2020 out-dez;60(4):45-48. 